\title{
A Cooperative Stochastic Model of Gene Expression
}

\author{
Siddhartha Roy ${ }^{1}$, Indrani Bose ${ }^{2}$ and Subhrangshu Sekhar Manna ${ }^{3}$ \\ ${ }^{1}$ Department of Biophysics, Bose Institute, P1/12, C.I.T. Scheme VII M, Calcutta-700054, India \\ ${ }^{2}$ Department of Physics, Bose Institute, 93/1, A.P.C. Road, Calcutta- 700009, India \\ 3 Satyendra Nath Bose National Centre for Basic Sciences, Block-JD, Sector-III, Salt Lake, Calcutta 700098, India
}

\begin{abstract}
Recent experiments at the level of a single cell have shown that gene expression occurs in abrupt stochastic bursts. Further, in an ensemble of cells, the levels of proteins produced have a bimodal distribution. In a large fraction of cells, the gene expression is either off or has a high value. We propose a stochastic model of gene expression the essential features of which are stochasticity and cooperative binding of RNA polymerase. The model can reproduce the bimodal behaviour seen in experiments.
\end{abstract}

Key Words: Gene expression. Stochastic transcription, Bimodal distribution

Gene expression is a fundamental and important biological process in a cell. Genes are part of DNA molecules and determine the structure of functional molecules such as RNAs and proteins. In each cell, at any instant of time, only a subset of genes present is active in directing RNA/protein synthesis. The gene expression is 'on' in such a case. The information present in the gene is expressed in the following manner. In the first step of gene expression, the sequence along one of the strands of the DNA molecule is copied or transcribed in a RNA molecule (mRNA). The sequence of mRNA molecules is then translated into the sequence of amino acids, which in turn determines the functional nature of the protein molecule produced. The rate and temporal sequence of gene expression is responsible for many aspects of biology. In the large majority of cases, the regulation of gene expression occurs at the level of transcription and hence an in-depth understanding of transcription regulation is a central focus of biology [1].

Recent experiments (see Appendix A), provide evidence that gene expression occurs in abrupt stochastic bursts at the level of an individual cell 20 4. Also, in many cases, in a population of cells the levels of proteins produced are distributed in a bimodal manner implying that in a large fraction of cells the gene expression is either off or has a high value [5]. In this paper, we propose a stochastic model of gene expression which provides a possible explanation of the observed bimodal behaviour.

Genes are transcribed into mRNA by an enzyme called RNA polymerase (RNAP). The process is initiated with the binding of RNAP to a site called promoter, usually near the beginning of the transcribed sequence. After the initial binding and subsequent conformational changes, the enzyme begins synthesis of the RNA chain and gradually translates along the DNA. The initial binding of RNAP to a promoter can be prevented by the binding of a regulatory protein $(\mathrm{R})$ to an overlapping segment of DNA (called operator) resulting in a turning off of mRNA production. There is a finite probability that the bound $\mathrm{R}$ molecule dissociates from the operator at any instant of time. RNAP molecule then has a certain probability of binding to the promoter and initiating transcription.

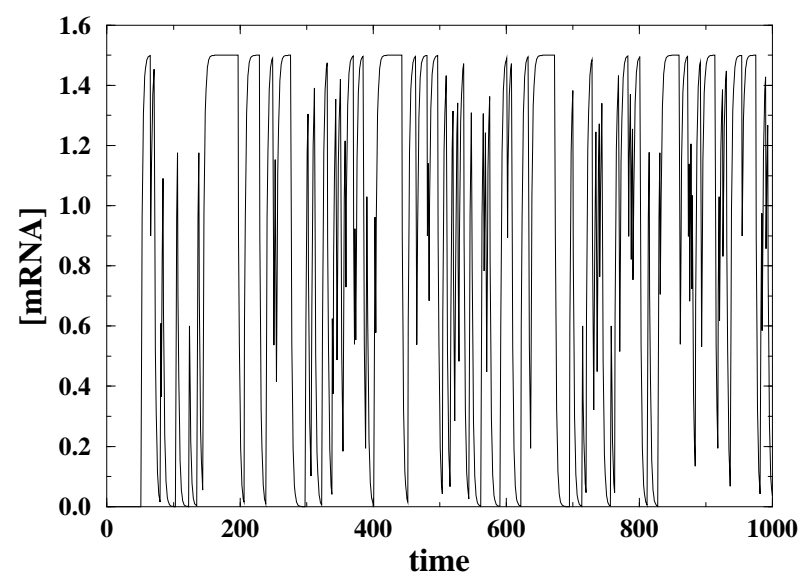

FIG. 1. Concentration of mRNA molecules [mRNA] in arbitrary units as a function of time $t$. The parameter values are $p_{1}=0.5, p_{2}=0.5, p_{3}=0.3, p_{4}=0.85, p_{5}=0.05$ and $\mu=0.4$.

Each of the possibilities described above actually involves a series of physico-chemical processes, a detailed characterization of which is not required for the model of gene expression that we propose here. We represent a gene by a one-dimensional lattice of $\mathrm{n}+2$ sites. The first two sites represent the operator and promoter respectively. The lattice is a coarse-grained description of an actual gene. In reality the operator and promoter regions may extend over a certain number of base pairs in the DNA and they can be overlapping or not. In our model they are represented as single sites. Each of the other sites in the lattice represents a finite number of base-pairs in the DNA molecule.

The different physico-chemical processes are lumped together into a few simple events which are random in nature. This lumping together avoids unnecessary complexity that has no bearing on the basic nature of the 
process. The operator $(\mathrm{O})$ and promoter $(\mathrm{P})$ together can be in four possible configurations : 10, 01, 00 and 11. The numbers ' 1 ' and ' 0 ' stand for 'occupied' and 'unoccupied'. The configuration $i j$ describes the occupation status of $\mathrm{O}(i)$ and $\mathrm{P}(j)$. For example, the configuration 10 corresponds to $\mathrm{O}$ being occupied by a $\mathrm{R}$ molecule and $\mathrm{P}$ being unoccupied. Similarly, in the configuration 01, $\mathrm{O}$ is unoccupied and $\mathrm{P}$ is occupied by a RNAP molecule. Binding of R and RNAP molecules are mutually exclusive so that the configuration 11 is strictly prohibited. Given a 00 configuration at time $t$, the transition probabilities to configurations 10 and 01 at time $t+1$ are $p_{1}$ and $p_{2}$ respectively. The probability of remaining in the configuration 00 is $1-p_{1}-p_{2}$. A 10 configuration at time $t$ goes to a 00 configuration at time $t+1$ with probability $p_{3}$ and remains unchanged with probability $1-p_{3}$.We have assumed all the probabilities to be time-independent. The justification for this approximation is that the number of free $\mathrm{R}$ and RNAP molecules in the cell are typically one or two orders of magnitude higher than the number of DNA sites they occupy. The RNAP molecule once bound to the promoter initiates transcription in the next time step, i.e., the 01 configuration makes a transition to a 00 configuration with probability 1 . The motion of RNAP is in the forward direction and the molecule covers a unit distance (the distance between two successive lattice sites) in each time step. Once the molecule reaches the last site of the lattice the transcription ends and a mRNA is synthesized.

The second major feature of our model is the cooperative binding of RNAP to the promoter, when an adjacent RNAP molecule is present. This implies that there is a higher probability of binding of RNAP to the promoter in one time step if another RNAP molecule is present at the site next to the promoter.In our model, the probability of cooperative binding of RNAP is $p_{4}$ which is larger than $p_{2}$. The probabilities $p_{1}$ and $1-p_{1}-p_{2}$ are changed to new values $p_{5}$ and $1-p_{4}-p_{5}$ respectively. Degradation of mRNA is taken into account by assuming the decay rate to be given by $\mu \mathrm{N}$ where $\mathrm{N}$ is the number of mRNAs present at time $t$. The number of $\mathrm{mR}$ NAs produced as a function of time is studied by Monte Carlo simulation.For the sake of simplicity, we have not tried to simulate protein levels or enzymatic products thereof, i.e., we study gene expression upto the level of transcription (mRNA synthesis). Since the number of protein molecules and converted products should be proportional to the mRNA present, no loss of generality is introduced by this simplification. The lattice consists of 52 sites $(n=50)$. Stochastic events are simulated with the help of a random number generator. The updating rule of our cellular automaton (CA) model is that in each time step $t$ the occupation status (0 or 1 ) of each site (except for the $\mathrm{O}$ site) at time $t-1$ is transferred to the nearest-neighbour site towards the right. If the $(n+2)$-th , i.e. , the last site is 1 at $t-1$, a mRNA is synthesized at $t$ and the number of mRNAs increases by 1 . In the same time step, the configuration $i j$ of OP is determined with the probabilities already specified. Thus in each time step, the RNAP molecule, if present on the gene, moves forward by unit lattice distance (progression of transcription) followed by the updating of the OP configuration. Figure 1 shows the concentration [mRNA] of mRNA molecules in the cell as a function of time for the parameter values $p_{1}=0.5, p_{2}=0.5, p_{3}=0.3, p_{4}$ $=0.85, p_{5}=0.05$ and $\mu=0.4$. Note that an almost four-fold increase in the probability of RNAP binding is assumed due to cooperativity. The stochastic nature of the gene expression is evident from the figure with random intervals between the bursts of activity. One also notices the presence of several bursts of large size. It is important to emphasize that the frequency of transitions between high and low expression levels is a function of the parameter values chosen and may be low for certain parameter values. For the probability values considered, the two predominantly favourable states are when the gene expression is off (state 1) and when a large amount of gene expression takes place (state 2). In the absence of RNAP, state 1 has greater weightage but with the chance binding of RNAP to the promoter (probability $p_{2}$ for this is small), the weight shifts to state 2 until another stochastic event terminates cooperative binding and the gene reverts to state 1 . The probability of obtaining a train of $N$ successive transcribing RNAP molecules is $p_{2} p_{4}^{N-1}\left(1-p_{4}\right)$. This is the geometric distribution function and the mean and the variance of the distribution are given by $p_{2} /\left(1-p_{4}\right)$ and $p_{2}\left(1+p_{4}-p_{2}\right) /\left(1-p_{4}\right)^{2}$ respectively.

For the probability values already specified, the simulation has been repeated for an ensemble of 3000 cells. For each cell, the time evolution is upto 10,000 time steps. Figure 2 shows the distribution of the number $N(m)$ of cells versus the fraction $m$ of the maximal number of mRNA molecules produced after 10,000 time steps. Two distinct peaks are seen corresponding to zero and maximal gene expression respectively. Such a bimodal distribution occurs over a wide range of parameter values. Figure 3 shows the distribution for parameter values $p_{1}=0.7, p_{2}=0.2, p_{3}=0.7, p_{4}=0.85, p_{5}=0.05$ and $\mu=0.5$. For the same set of parameter values but with $p_{3}=0.1$, the bimodal distribution is lost and one gets a single prominent peak corresponding to maximal gene expression. Distributions with several peaks of random heights are obtained when the parameter values do not produce the effect. The full parameter space describing the three different regions of unimodal, bimodal and multi-peak distributions has not been explored in detail as yet. The transition from one region to another is in a broad sense like a phase transition. Since the distribution of transcribing RNAPs is bimodal in nature, many results like the distribution of time intervals in between bursts of gene expression can be written down from the 
stochastic theory of such distributions [6].

In summary, we have proposed a stochastic model which can reproduce the bimodal distribution in gene expression observed in recent experiments. We have suggested that the stochastic nature of transcription coupled with RNAP binding cooperativity may result in discontinuous levels of gene expression and consequent bimodal distribution of expressed protein levels, as observed in a number of experiments. To our knowledge, no stochastic mechanism of bimodal distribution has been offered so far. Increasing emphasis on the stochastic nature of the developmental switches operating at the level of transcription suggests that the bimodal distribution of protein levels may have a role to play in such mechanisms [7.

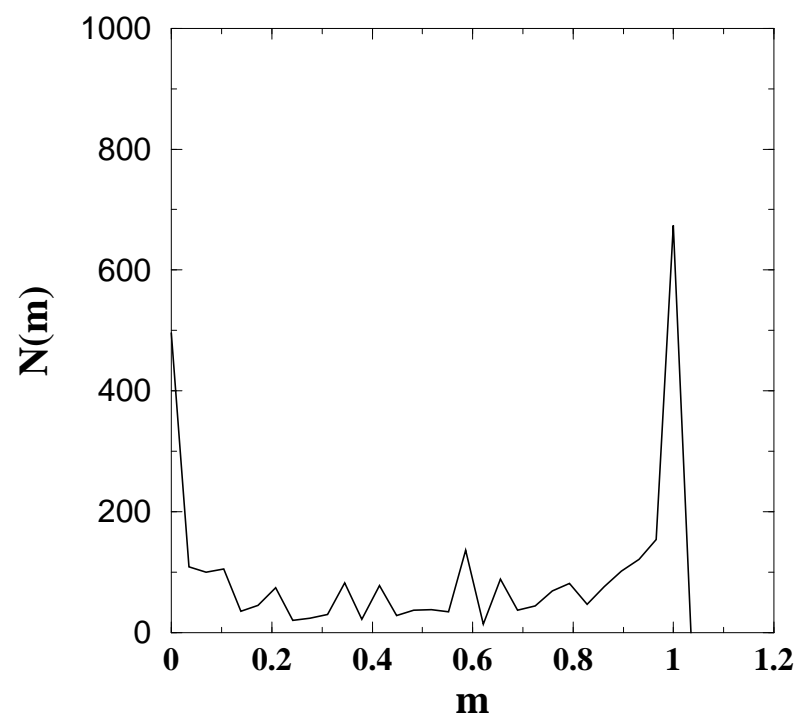

FIG. 2. Distribution of no. $\mathrm{N}(\mathrm{m})$ of cells expressing fraction $\mathrm{m}$ of maximal number of mRNA after 10,000 time steps. The total number of cells is 3000 . The parameter values are $p_{1}=p_{2}=0.5, p_{3}=0.3, p_{4}=0.85, p_{5}=0.05$ and $\mu=0.4$.

\section{Appendix A}

In this Appendix, we discuss the various biological aspects of the problem studied in this paper.

Biological variability is a product of interaction of genes with the environment. With the advent of rapid genome sequencing methods and remarkable success in sequencing genomes from many organisms, the thrust is now gradually shifting to the functional aspects of information present in the genome. The genome of an organism is a storehouse of sequential information contained in all the genes specific to that organism. Through gene expression, the sequential information determines the structure of functional molecules like RNAs and proteins.Since the advent of molecular biology, the regulation of gene expression has been studied in solution or in an ensemble of cells where an average property is measured. This mode of study was necessary, as it was difficult to obtain information at the level of an individual cell. A complete understanding of cellular processes, however, needs an appreciation of events at the level of an individual cell and extrapolation to an ensemble of cells.

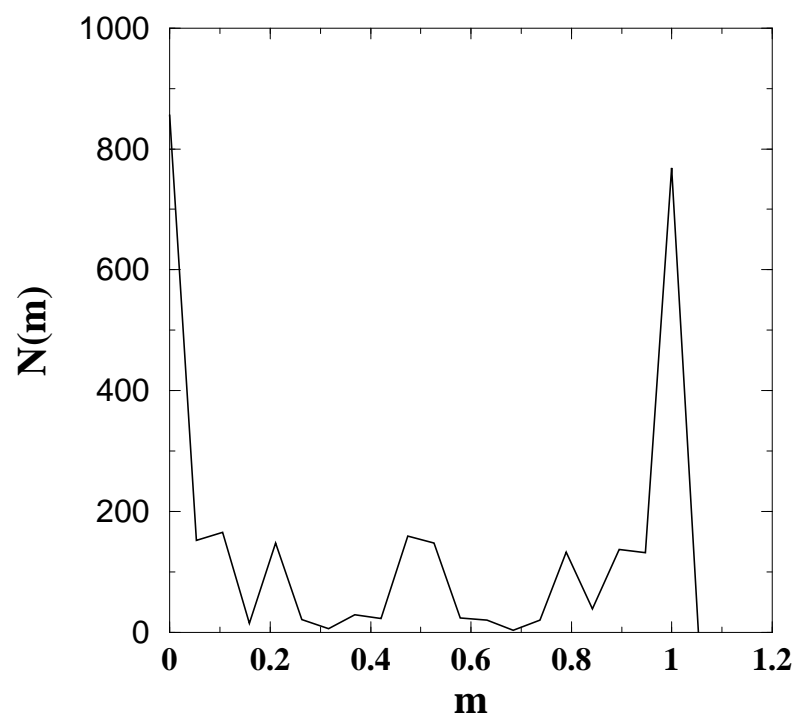

FIG. 3. Distribution of no. $\mathrm{N}(\mathrm{m})$ of cells expressing fraction $\mathrm{m}$ of maximal number of mRNA after 10,000 time steps. The total number of cells is 3000 . The parameter values are $p_{1}=0.7, p_{2}=0.2, p_{3}=0.7, p_{4}=0.85, p_{5}=0.05$ and $\mu=0.5$.

Recent advances have made it possible to study processes within a single cell unmasked by ensemble averaging 8]. The simplest event one can study at the individual cell level is that of the expression of a reporter gene such as lacZ and GFP. In the former case, the end product is an enzyme $\beta$ - galactosidase which is capable of hydrolyzing a non-coloured substrate to a coloured product. In the latter case the protein itself is fluorescent. Hence, the gene expression can be directly studied either colorimetrically or flurometrically at the level of an individual cell. Recent experiments using such techniques, provide evidence that gene expression occurs in abrupt stochastic bursts at the level of an individual cell [2 4 . The stochastic nature of gene expression is also evident when levels of $\beta$-galactosidase were examined in an ensemble of cells. Levels of $\beta$-galactosidase are distributed in a bimodal manner, in a large fraction of cells the gene expression is either off or has a high value [5].

Some theories have been proposed so far to explain the so-called 'all or none' phenomenon in gene expression. These theories are mostly based on an autocatalytic feedback mechanism, synthesis of the gene product gives rise to the transport or production of an activator molecule [5,8,9]. While such processes are certainly 
possible, the bimodal distribution is a much more general phenomenon and has now been found in many types of cells, from bacterial to eukaryotic and for different types of promoters 2 . 4 .

The two major features of the model of gene expression that we have proposed in this paper are stochasticity and cooperative binding of RNA polymerase. As already explained in the paper, the different physico-chemical processes associated with gene expression are lumped together into a few simple events which are random in nature. To give an example, for many prokaryotic promoters, there is a two-step reaction scheme in which a RNAP open complex is formed preceded by the formation of a closed complex. RNAP initiates transcription only from the open complex. The isomerization step is rate-limiting in many cases [10]. We define the on-rate of RNAP as the composite of several steps with the final attainment of the open complex. The cooperative binding of RNAP to the promoter in our model implies that there is a higher probability of binding of RNAP to the promoter in one time step if another RNAP molecule is present at the site next to the promoter. Although such binding cooperativity has not been studied in prokaryotic polymerases, it has been demonstrated in polio-virus RNA-dependent RNA polymerase [11]. Cooperative binding of proteins to DNA is now well established. In most cases of regulatory proteins, the binding cooperativity is mediated through protein-protein interaction although increasing evidence of DNA mediated effects are being reported 112. In the case of RNAP binding to promoters, however, there are now widespread reports of transcription generated increase in negative supercoiling with consequent increase in rate of transcription 13, 14. In many promoters, the transcription initiation is sensitive to the supercoiling status of the DNA. It has been reported that transcription generates increased negative supercoiling through several hundred base pairs [15]. Thus it is entirely plausible and likely that active transcription downstream of the promoter site may lead to increased binding of RNAP and open-complex formation. One can also envisage other mechanisms for generating this kind of cooperativity. For example, if the polymerase-generated negative supercoiling (after initial movement) inhibits binding of the repressor, it would effectively increase polymerase binding probability.

Transcription is one of the most important events in the life-cycle of a cell. The temporal sequence of events occurring during transcription is of utmost importance in its understanding and has been studied extensively.
The general description of transcription as well as other cellular events have tended to be deterministic in nature. In the cell there are only a few DNA molecules and a few molecules of free RNAP. It is likely that the number of molecules in the cell is not high enough so that a deterministic description of this small ensemble is correct. At the level of a single cell, probabilistic descriptions are more appropriate. Increasingly, probabilistic descriptions of cellular events, including transcription are being offered [16 18].

Electronic addressies: sidroy@vsnl.com, indrani@boseinst.ernet.in,manna@boson.bose.res.in

[1] Genes V by B. Levin (Oxford University Press, New York 1994)

[2] G. Zlokarnik, P.A. Negulescu, T.E. Knapp, L. Mere, N. Burres, L. Feng, M. Whitney, K. Roemer and R.Y. Tsien, Science 279, 84 (1998)

[3] P. A. Negulescu, N. Shastri and M.D. Cahalan, Proc. Natl. Acad. Sci. 91, 2873 (1994)

[4] J. Karttunen and N. Shastri, Proc. Natl. Acad. Sci. 88, 3972 (1991)

[5] A. Novick and M. Weiner, Proc. Natl. Acad. Sci. 43, 553 (1957)

[6] An Introduction to Probability Theory and its Applications by W.Feller (Wiley Eastern Limited 1984)

[7] D.A. Hume, Blood 96, 2323 (2000)

[8] M.T.Beckman and K. Kirkegaard, J. Biol. Chem. 273, 6724 (1998)

[9] T.A. Carrier and J.D. Keasling, J. Theor. Biol. 201, 25 (1999)

[10] W.R. McClure, C.L. Cech and D.E. Johnston, J.Biol. Chem. 253, 8941 (1978)

[11] D. A. Siegele and J.C. Hu, Proc. Natl. Acad. Sci. 94, 8168 (1997)

[12] S. Adhya, Ann. Rev. Genetics 23, 227 (1989)

[13] K.Y. Rhee, M. Opel, E. Ito, S. Hung, S.M. Arfin and G.W. Hatfield, Proc. Natl. Acad. Sci. 96, 14294 (1999)

[14] S.D. Sheridan, C.J. Benham and G. W. Hatfield, J. Biol. Chem. 273, 21298 (1998)

[15] A.S. Krasilnikov, A. Podtelezhnikov, A. Vologodskii and S.M. Mirkin, J. Mol. Biol. 292, 1149 (1999)

[16] H.H.Mcadams and A. Arkin, Proc. Natl. Acad. Sci. 94, 814 (1997)

[17] H.H. McAdams and A. Arkin, Trends in Genetics 15, 65 (1999)

[18] A.M.Kierzek,J.Zaim and P.Zielenkiewicz, preprint 